\title{
Mutation or loss of Wilms' tumor gene 1 (WT1) are not major reasons for immune escape in patients with AML receiving WT1 peptide vaccination
}

\author{
Antonia Busse ${ }^{1 *}$, Anne Letsch ${ }^{1}$, Carmen Scheibenbogen ${ }^{2}$, Anika Nonnenmacher ${ }^{1}$, Sebastian Ochsenreither ${ }^{1}$, \\ Eckhard Thiel', Ulrich Keilholz'
}

\begin{abstract}
Background: Efficacy of cancer vaccines may be limited due to immune escape mechanisms like loss or mutation of target antigens. Here, we analyzed $10 \mathrm{HLA}$-A2 positive patients with acute myeloid leukemia (AML) for loss or mutations of the WT1 epitope or epitope flanking sequences that may abolish proper T cell recognition or epitope presentation.

Methods: All patients had been enrolled in a WT1 peptide phase II vaccination trial (NCT00153582) and ultimately progressed despite induction of a WT1 specific T cell response. Blood and bone marrow samples prior to vaccination and during progression were analyzed for mRNA expression level of WT1. Base exchanges within the epitope sequence or flanking regions (10 amino acids $\mathrm{N}$ - and C-terminal of the epitope) were assessed with melting point analysis and sequencing. HLA class I expression and WT1 protein expression was analyzed by flow cytometry.
\end{abstract}

Results: Only in one patient, downregulation of WT1 mRNA by 1 log and loss of WT1 detection on protein level at time of disease progression was observed. No mutation leading to a base exchange within the epitope sequence or epitope flanking sequences could be detected in any patient. Further, no loss of HLA class I expression on leukemic blasts was observed.

Conclusion: Defects in antigen presentation caused by loss or mutation of WT1 or downregulation of HLA molecules are not the major basis for escape from the immune response induced by WT1 peptide vaccination.

\section{Background}

Over-expression of Wilms' tumor gene 1 (WT1) is present in a variety of malignant tumors, including acute leukemias [1-3] and a variety of solid neoplasms [4]. The WT1 protein is a transcription factor critically involved in tumor cell proliferation, making it a suitable target for therapeutic strategies including vaccine approaches [5]. Clinical vaccination trials with WT1 peptides and protein in AML/MDS have been recently initiated leading to the induction of epitope specific cytotoxic $\mathrm{T}$ cells and unprecedented clinical efficacy [6-8]. However, even in case of induction of a robust $\mathrm{T}$ cell response cancer vaccines in

\footnotetext{
* Correspondence: antonia.busse@charite.de

${ }^{1}$ Charité - CBF, Department of Medicine III, Berlin, Germany
}

general have only limited efficacy. Several immune escape mechanisms have been identified [9-11]. Important escape mechanisms on tumor cell site are loss or downregulation of tumor associated antigens (TAA) and mutation of TAA $[12,13]$. A mutation within the sequence of an epitope may abolish proper HLA class I binding, T cell recognition or proteasomal processing. Another less recognised mechanism interfering with antigen presentation may be a mutation of the flanking sequence of an epitope that may prohibit or decrease processing of the epitope by the proteasome or extraproteasomal proteases $[14,15]$. In addition, antigen presentation can be distorted by mechanisms such as decrease in HLA class I expression [16-18] or alterations in the antigen processing pathway [19-21]. 
Here we address loss or mutation of WT1 as a potential immune evasion mechanism in patients from a clinical phase II trial of WT1 peptide vaccination in acute myeloid leukaemia (AML).

\section{Methods}

Patients

Patients were treated within a phase II vaccination trial (NCT00153582) [8] and received sequential vaccinations with the HLA-A2-restricted WT1 126-134 peptide + KLH and GM-CSF as adjuvants. Detailed patient characteristics are previously published in Keilholz et al 2009: patient no 1 ; no 4 ; no 5 ; no 8 ; no 9 ; no 11 ; no 12 ; no 13; no 15; one patient is not published yet) [8]. All patients gave written informed consent to participate in the study according to the Declaration of Helsinki. The study was approved by the local ethics board.

\section{Blood and bone marrow samples}

Bone marrow and peripheral blood samples have been collected before vaccination and during progression in heparinized tubes and mononuclear cells (MNCs) were isolated by Ficoll Isopaque density gradient centrifugation (Pharmacia, Germany).

\section{mRNA extraction and reverse transcription}

Samples were resuspended in guanidium thiocyanate (GTC) buffer and stored at $-80^{\circ} \mathrm{C}$. Further processing of samples was performed as previously described [22]. In brief, total RNA was isolated by RNeasy Mini Kit including RNase-Free DNase Set (Qiagen, Germany) according to the manufactures recommendations. For reverse transcription, Omniscript Reverese Transcriptase kit (Qiagen, Germany) was used.

\section{Quantification of WT1 expression levels}

Quantitative Real Time RT-PCR assays were performed using a LightCycler (Roche Diagnostics) with specific primers for WT1 and the housekeeping gene porphobilinogen deaminase (PBGD) as described elsewhere [22]. For quantification, PCR products generated from WT1 cDNAs and from porphobilinogen deaminase (PBGD) cDNAs were cloned into the vector pCR2.1TOPO (Invitrogen, The Netherlands). A standard curve with 3 dilutions of the appropriate plasmid in duplicates was included in each PCR run. Analysis of RT-PCR expression data was performed with the LightCycler software (version 3). Crossing points were assessed by the second derivate maximum algorithm and plotted against the concentrations of the standards. Sample concentrations were calculated using the plasmid standard curve resulting in marker concentrations. All samples were analysed in duplicate. The average value of both duplicates was used as a quantitative value. To correct for differences of cDNA amount on a per-sample basis, results were provided as ratio to $\mathrm{PBGD}$ expression.

\section{Mutation analysis}

Base exchanges within the epitope sequence or epitope flanking sequences (10 amino acids $\mathrm{N}$ - and C-terminal of the epitope) were analyzed with melting point analysis after amplification with the specific primers WT1 Mut fw 5'-TGTCCACTTTTCCGGC-3' and WT1 Mut rev 5'-GTCCCGTCGAAGGTGA-3 on a LightCycler instrument. To cover the whole sequence 2 wild-type complementary detection probe pairs were used $(P$, dephosphorylated; X, Fluorescein; Y, LC Red 640): probe pair 1: 5'-Y GCGCGTTAGGAAACATCCTGG P, 5'TGGCCGGATGACGCCTGG X, probe pair 2: 5' $-Y$ CTGGGCAGGTAGGGC P, 5’-TTAGGAAACATCCTG GCCTGGCCG X. To confirm the results obtained by melting curve analysis sequencing was performed in 4 patients.

\section{Flow cytometry}

For determination of HLA Class I expression and HLAA2 expression, leukemic blasts were stained with FITC conjugated anti-HLA class I monoclonal antibody (mAb) B9.12.1 (Beckmann Coulter) and with Alexa 647 conjugated mAb anti-HLA-A2 BB7.2 (AbSerotec) respectively. For exclusion of monocytes and lymphocytes samples were additionally stained with PerCP conjugated anti CD3 $\mathrm{mAb}$ and anti CD14 mAb (both BD Bioscience) and for exclusion of dead cells the LIVE/ DEAD Fixable Violet Dead Cell Stain Kit (Molecular Probes) was used. For detection of WT1 expression in leukemic blasts extracellular staining was done with PEconjugated mAb against CD34 (Becton Dickinson) and intracellular staining with mAbs against WT1 (clone 6F$\mathrm{H} 2$, Dako) as primary antibody and goat anti mouse (GAM)-FITC (JacksonImmunoResearch) as secondary antibody. T cell response assessment was carried out as described in detail in Keilholz et al 2009 [8]. A cytokine response was considered positive if the percentage of WT1-peptide-specific cytokine producing CD3+CD8+T cells was at least 2-fold the percentage of cytokine producing $\mathrm{CD} 3+\mathrm{CD} 8+\mathrm{T}$ cells in response to an HIV control peptide; a tetramer response was considered positive if the frequency of tetramer positive CD3+CD8+ T cells exceeded $0.3 \%$, which was the mean +2 standard deviations $(0.16 \%+0.14 \%)$ observed in 12 healthy control subjects.

Data acquisition was performed on a FACSCalibur (Becton Dickinson) and data were analyzed using CellQuest software.

\section{Results and Discussion}

Ten HLA-A2 positive patients with AML were analysed for mRNA expression levels of WT1 and for mutations of the WT1 epitope or epitope flanking sequences. All patients had received sequential vaccinations with the HLA-A2-restricted WT1 126-134 peptide with adjuvants 
within a phase II vaccination trial and progressed after an initial interval of vaccine efficacy [8]. T cell response to vaccination was analyzed as previously published in Keilholz et al 2009 (patient no 1; no 4; no 5; no 8; no 9; no 11; no 12; no 13; no 15; one patient is not published yet) [8]: In 8 of these 10 patients WT1 126-134 tetramer $+\mathrm{T}$ cells during the course of vaccination were found in peripheral blood (mean percentage of WT1 126-134 tetramer + cells in the CD3+ CD8 + $\mathrm{T}$ cell population $0.76 \%$ [0.3\%-1.09\%]). Moreover, to analyze the functional activity of WT1 126-134 specific T cells raised by vaccination, the reactivity of $\mathrm{CD} 3+\mathrm{CD} 8+\mathrm{T}$ cells against WT1 126-134 peptide loaded cells was measured by intracellular IFN- $\gamma$ and/or TNF- $\alpha$ cytokine staining. In 8 of 10 patients the presence of TNF- $\alpha$ and/or IFN- $\gamma$ producing WT1 126-134 specific CD3+CD8+ T cells could be induced by vaccination. 7 patients showed a TNF- $\alpha$ response with a mean percentage of TNF- $\alpha+$ cells in the CD3+CD8+ T cell population of $0.26 \%(0.1 \%-0.6 \%)$ and 7 patients showed a IFN- $\gamma$ response with a mean percentage of IFN $-\gamma+$ cells in the CD $3+\mathrm{CD} 8+\mathrm{T}$ cell population of $0.23 \%(0.09-0.6 \%)$. However, one patient showed no WT1 126-134 tetramer + T cells or cytokine response in peripheral blood.

As loss or downregulation of tumor antigens are potential immune escape mechanisms [12,13], first bone marrow samples obtained before vaccination and during progression were analyzed for expression levels of WT1 by real-time RT-PCR. In 9 out of 10 patients bone marrow WT1 levels were constant or increased at the time point of progression mirroring the kinetics of bone marrow blasts during treatment (figure 1). In one patient, however, down-regulation of WT1 by $1 \log$ was observed although bone marrow blasts reached the same level at time of disease progression as before vaccination. In this latter patient WT1 protein was undetectable by intracellular flow cytometry at time of disease progression, consistent with downregulation of WT1 mRNA and protein as escape mechanism in this single patient.

WT1 mutations have been reported in about $10 \%$ of patients with AML [23]. They were frequently observed in DNA binding portions of the WT1 protein and may therefore contribute to leukemogenesis [24]. However, mutations have also been observed in exon 1 , affecting the epitope flanking regions of the WT1 126-134 epitope [23]. Mutations of the epitope sequence or its flanking sequences could represent a possible immune escape mechanism as they may abolish proper HLA class I binding, T cell recognition or proteasomal processing. Therefore, mutation analysis was performed in both samples obtained before vaccination and during progression. However, no mutation leading to a base exchange within the epitope sequence or epitope flanking sequences could be detected in any patient. As

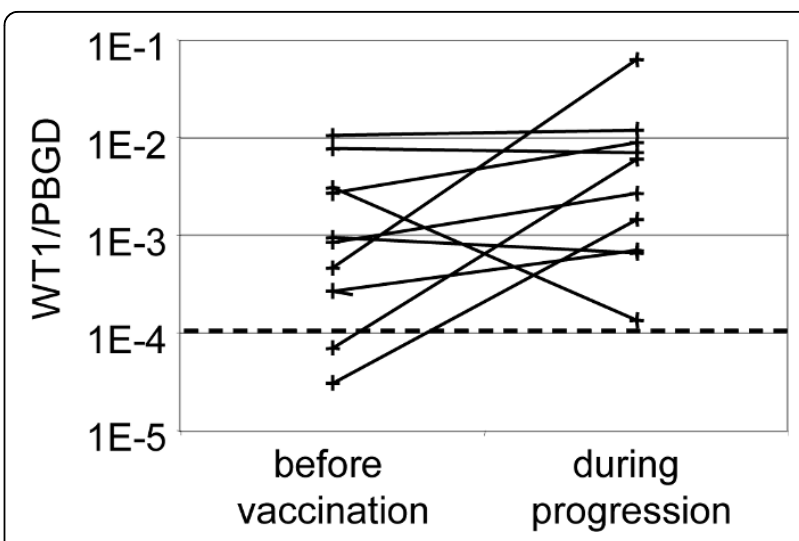

Figure 1 WT1 expression levels before vaccination and during progression. The relative amount was expressed as ratio WT1 [pg/ $\mu l] / P B G D[p g / \mu l])$. Dotted line: normal bone marrow cut-off level.

expected, the known single nucleotide polymorphism $\mathrm{C} /$ T (NM_000378, mRNA position 790, coding position 3, protein residue Asn) at amino acid position 130 was observed in 6 patients.

To exclude failure of vaccine efficacy due to HLA class I downregulation [16-18,25], cell surface HLA class I expression on leukemic blasts was analyzed in 9 patients by flow cytometry during progression. The median percentage of HLA class I expressing blasts was 96\% (84\%-99\%). Compared to HLA class I expression on blasts before therapy ( 5 patients analyzed) there was no significant downregulation. To exclude selective loss of the HLA-A2 allele, we analyzed HLA-A2 expression on leukemic blasts of 5 patients. In all 5 patients more than $90 \%$ of blasts stained positive for HLA-A2. In none of the patients a difference of HLA-A2 expression before therapy and at the time point of progression was observed.

\section{Conclusions}

We have no evidence for an immune escape due to loss or mutation of WT1 or HLA class I downregulation as has been reported for immunotherapy targeting differentiation antigens in melanoma [12]. This finding supports the use of tumor target antigens like WT1 which are crucial for tumor cell proliferation. However, further studies, especially on mechanisms of immune evasion at the effector phase of the anti-tumor immune response, are indicated to determine potential inhibitory immune mechanisms during WT1 peptide vaccination.

\section{Acknowledgements}

We thank David Stather for technical help.

\section{Author details}

'Charité - CBF, Department of Medicine III, Berlin, Germany. ${ }^{2}$ Institute of Medical Immunology, Charité - CCM, Berlin, Germany. 


\section{Authors' contributions}

$A B$ has made substantial contributions to conception and design, acquisition of data, analysis and interpretation of data and wrote the manuscript; AL, AN and OS have made substantial contributions to acquisition of data, analysis and interpretation of data. CS have been involved in conception and design, interpretation of data and revising the manuscript critically for important intellectual content, ET has made substantial contributions to conception and design and was involved in revising the manuscript critically for important intellectual content, UK: has made substantial contributions to conception and design, as well as analysis and interpretation of data and wrote the manuscript.

All authors have read and approved the final manuscript.

\section{Competing interests}

Supported by a grant from the from the José-Carreras Leukemia Foundation and from the "Stiftung zur Bekaempfung der Leukaemie"

Received: 1 September 2009

Accepted: 21 January 2010 Published: 21 January 2010

\section{References}

1. Inoue K, Ogawa H, Sonoda Y, Kimura T, Sakabe H, Oka Y, Miyake S, Tamaki $\mathrm{H}$, Oji $Y$, Yamagami T, et al: Aberrant overexpression of the Wilms tumor gene (WT1) in human leukemia. Blood 1997, 89:1405-1412.

2. Menssen HD, Renkl HJ, Rodeck U, Maurer J, Notter M, Schwartz S, Reinhardt R, Thiel E: Presence of Wilms' tumor gene (wt1) transcripts and the WT1 nuclear protein in the majority of human acute leukemias. Leukemia 1995, 9:1060-1067.

3. Miwa H, Beran M, Saunders GF: Expression of the Wilms' tumor gene (WT1) in human leukemias. Leukemia 1992, 6:405-409.

4. Nakatsuka S, Oji Y, Horiuchi T, Kanda T, Kitagawa M, Takeuchi T, Kawano K, Kuwae $Y$, Yamauchi A, Okumura M, et al: Immunohistochemical detection of WT1 protein in a variety of cancer cells. Mod Pathol 2006, 19:804-814.

5. Sugiyama H: Cancer immunotherapy targeting WT1 protein. Int I Hematol 2002, 76:127-132.

6. Oka Y, Tsuboi A, Oji Y, Kawase I, Sugiyama H: WT1 peptide vaccine for the treatment of cancer. Curr Opin Immunol 2008, 20:211-220.

7. Rezvani K, Yong AS, Mielke S, Savani BN, Musse L, SUperata J, Jafapour B, Boss C, Barrett AJ: Leukemia-associated antigen-specific T-cell responses following combined PR1 and WT1 peptide vaccination in patients with myeloid malignancies. Blood 2008, 111:236-242.

8. Keilholz U, Letsch A, Busse A, Asemissen AM, Bauer S, Blau IW, Hofmann WK, Uharek L, Thiel E, Scheibenbogen C: A clinical and immunologic phase 2 trial of Wilms tumor gene product 1 (WT1) peptide vaccination in patients with AML and MDS. Blood 2009, 113:6541-6548.

9. Gajewski TF, Meng Y, Harlin H: Immune suppression in the tumor microenvironment. J Immunother 2006, 29:233-240.

10. Seliger B: Strategies of tumor immune evasion. BioDrugs 2005, 19:347-354.

11. Kim R, Emi M, Tanabe K: Cancer immunoediting from immune surveillance to immune escape. Immunology 2007, 121:1-14.

12. Slingluff CL Jr, Colella TA, Thompson L, Graham DD, Skipper JC, Caldwell J, Brinckerhoff L, Kittlesen DJ, Deacon DH, Oei C, et al: Melanomas with concordant loss of multiple melanocytic differentiation proteins: immune escape that may be overcome by targeting unique or undefined antigens. Cancer Immunol Immunother 2000, 48:661-672.

13. Singh R, Paterson Y: Immunoediting sculpts tumor epitopes during immunotherapy. Cancer Res 2007, 67:1887-1892.

14. Seifert U, Liermann H, Racanelli V, Halenius A, Wiese M, Wedemeyer $H$, Ruppert T, Rispeter K, Henklein P, Sijts A, et al: Hepatitis C virus mutation affects proteasomal epitope processing. J Clin Invest 2004, 114:250-259.

15. Theobald M, Ruppert T, Kuckelkorn U, Hernandez J, Haussler A, Ferreira EA Liewer U, Biggs J, Levine AJ, Huber C, et al: The sequence alteration associated with a mutational hotspot in p53 protects cells from lysis by cytotoxic T lymphocytes specific for a flanking peptide epitope. J Exp Med 1998, 188:1017-1028.

16. Marincola FM, Jaffee EM, Hicklin DJ, Ferrone S: Escape of human solid tumors from T-cell recognition: molecular mechanisms and functional significance. Adv Immunol 2000, 74:181-273.

17. Algarra I, Cabrera T, Garrido F: The HLA crossroad in tumor immunology. Hum Immunol 2000, 61:65-73.
18. Watson NF, Ramage JM, Madjd Z, Spendlove I, Ellis IO, Scholefield JH, Durrant LG: Immunosurveillance is active in colorectal cancer as downregulation but not complete loss of MHC class I expression correlates with a poor prognosis. Int I Cancer 2006, 118:6-10.

19. Seliger B, Atkins D, Bock M, Ritz U, Ferrone S, Huber C, Storkel S: Characterization of human lymphocyte antigen class I antigenprocessing machinery defects in renal cell carcinoma lesions with special emphasis on transporter-associated with antigen-processing down-regulation. Clin Cancer Res 2003, 9:1721-1727.

20. Seliger B, Maeurer MJ, Ferrone S: Antigen-processing machinery breakdown and tumor growth. Immunol Today 2000, 21:455-464.

21. Garcia-Lora A, Martinez M, Algarra I, Gaforio JJ, Garrido F: MHC class Ideficient metastatic tumor variants immunoselected by $T$ lymphocytes originate from the coordinated downregulation of APM components. Int $J$ Cancer 2003, 106:521-527.

22. Siehl JM, Thiel E, Heufelder K, Snarski E, Schwartz S, Mailander V, Keilholz U: Possible regulation of Wilms' tumour gene 1 (WT1) expression by the paired box genes PAX2 and PAX8 and by the haematopoietic transcription factor GATA-1 in human acute myeloid leukaemias. $\mathrm{Br} J$ Haematol 2003, 123:235-242.

23. King-Underwood L, Pritchard-Jones K: Wilms' tumor (WT1) gene mutations occur mainly in acute myeloid leukemia and may confer drug resistance. Blood 1998, 91:2961-2968.

24. Summers K, Stevens J, Kakkas I, Smith M, Smith LL, Macdougall F, Cavenagh J, Bonnet D, Young BD, Lister TA, Fitzgibbon J: Wilms' tumour 1 mutations are associated with FLT3-ITD and failure of standard induction chemotherapy in patients with normal karyotype AML. Leukemia 2007, 21:550-551, author reply 552.

25. Chang CC, Ferrone S: Immune selective pressure and HLA class I antigen defects in malignant lesions. Cancer Immunol Immunother 2007, 56:227236.

\section{doi:10.1186/1479-5876-8-5}

Cite this article as: Busse et al:: Mutation or loss of Wilms' tumor gene 1 (WT1) are not major reasons for immune escape in patients with AML receiving WT1 peptide vaccination. Journal of Translational Medicine 2010 8:5.

\section{Submit your next manuscript to BioMed Central and take full advantage of:}

- Convenient online submission

- Thorough peer review

- No space constraints or color figure charges

- Immediate publication on acceptance

- Inclusion in PubMed, CAS, Scopus and Google Scholar

- Research which is freely available for redistribution 